\title{
How to Become a Competitive Applicant for Gastroenterology Fellowship: Tips and Tricks for Success Part II
}

\author{
Nikki Duong ${ }^{1} \cdot$ Elizabeth S. Aby ${ }^{2} \cdot$ Kelly E. Hathorn ${ }^{3} \cdot$ C. Roberto Simons-Linares ${ }^{4} \cdot$ Mohammad Bilal $^{5}$
}

Published online: 30 April 2020

(c) Springer Science+Business Media, LLC, part of Springer Nature 2020

\begin{abstract}
In recent years, gastroenterology has become one of the most competitive subspecialties included in the internal medicine fellowship match, which increases the stressful nature of an already potentially nerve-wracking process for the trainee. Though each applicant has unique strengths and thus the process is somewhat individualized, there are some basic principles that can render an applicant more competitive for a gastroenterology fellowship. These include establishing mentorship, achieving scholarly work, building your resume, honing interpersonal and networking skills, and writing a well-planned application. The goal of this article is to outline some basic principles that will help improve the competitiveness of an applicant, and also highlight some practical tips and tricks for applicants with diverse backgrounds, such as international medical graduates, minority applicants, and women in medicine.
\end{abstract}

\section{Introduction to Part II}

In the second part of our two part series entitled "How to become a competitive applicant for gastroenterology fellowship: Tips and Tricks for Success," we discuss how you can effectively build your resume, develop a brand and reputation, address issues pertaining to women applicants and minorities, and provide practical suggestions regarding the fellowship application process.

Nikki Duong

Nduong91@gmail.com

1 Department of Internal Medicine, Georgetown University Hospital, 3800 Reservoir Rd NW, Washington, DC 20007, USA

2 Division of Gastroenterology, Hepatology and Nutrition, University of Minnesota, 500 SE Harvard St, Minneapolis, MN 55455, USA

3 Division of Gastroenterology, Hepatology and Endoscopy, Brigham and Women's Hospital, Harvard Medical School, 75 Francis Street, Boston, MA 02115, USA

4 Department of Gastroenterology, Hepatology and Nutrition, Cleveland Clinic, 9500 Euclid Avenue, Cleveland, OH 44195, USA

5 Division of Gastroenterology, Hepatology and Nutrition, Beth Israel Deaconess Medical Center, Harvard Medical School, 330 Brookline Avenue, Boston, MA 02215, USA

\section{Building Your Resume}

As mentioned in Part I, although scholarly work is an integral part of your application, there are other factors that go into building an excellent resume. Since programs view your resume/application in the format that the Electronic Residency Application Service (ERAS) designs, the layout may differ from how you had previously formatted your resume. Though you might remember this format from your residency application, and can use this as a template, it is important to study the application format prior to building your resume so that you can know what is important to highlight, and where in the application to do so.

There are several other strategies to build your resume that can accentuate the strength of your application. One thing to consider is the utility (and feasibility) of pursuing an away rotation at another institution, as discussed in Part I. Volunteering and being active in community or medical work outside of your clinical work is also a great way to build your resume. Several organizations such as the American College of Physicians (ACP), Crohn's and Colitis Foundation (CCF), and the American Liver Foundation (ALF) have many volunteering events, which can also serve as superb networking opportunities. We certainly advise becoming members of these organizations in order to stay informed of opportunities in your area. Similarly, being a member of the national organizations such as the ACG, AGA and AASLD can yield certain opportunities. The AGA 
has a "Young Delegate" program that often has wonderful volunteering and experience-building opportunities for trainees [1]. The AASLD has opportunities to be an AASLD emerging liver scholar or ambassador, enabling you to create your own activity for other likeminded trainees. Also, being part of your institution's committees can be a great learning experience that also helps to strengthen your application.

\section{Building Your Brand}

The old saying goes, "to be a great consultant, you need to be a great internist first." Hence, to be a competitive applicant, you first need to be an exceptional resident, defined as being kind, compassionate, clinically astute, hardworking, and most importantly, demonstrating a well-developed air of professionalism. It is essential that you build a reputation of being reliable and respected by your peers. Research projects can sometimes carry additional stress, but the golden rule is to never compromise your clinical care and reputation as a so-called "great resident" because you do not want to jeopardize your research activities.

During your training, it is important to show your interest in GI by taking advantage of electives in GI, attending your institution's GI conferences, and building relationships with fellows and faculty. Becoming actively involved in the GI community not only enables familiarity with faculty and fellows, but also provides access to valuable advice and informal mentoring.

In the current era, social media is a great tool for networking and building your reputation. For instance, there has been significant growth of the number of GI physicians on Twitter, providing opportunities for networking, as posting well thought out academic and scientific material and engaging in conversations can improve your visibility, which could improve your attractiveness as a fellowship applicant. Needless to say, as with an away rotation, social media can also negatively impact your reputation, and so should be used cautiously [2].

\section{Women/Minorities}

In a 2012 ASGE survey of member self-identification, $<10 \%$ of practicing gastroenterology providers were underrepresented minorities and only 1 in 10 gastroenterologists were women [3]. Despite the promotion of diversity and inclusion among many fellowship programs, some programs have a more developed support infrastructure than do others. For example, Sethi et al. presented data at DDW 2019 where they demonstrated that the proportion of women within a specific fellowship program is significantly higher if either the program director or associate director is a woman (4.26 vs. 3.36, $p=0.041)$. Moreover, if any department leader is a female, the difference is even greater (4.04 vs. 2.87, $p=0.007$ [4]. During early training, consider connecting with a mentor from another institution through the American College of Gastroenterology (ACG)'s mentorship program; these connections may help provide insight into the culture at different fellowship programs. At national meetings, network with fellows at programs in which you may be interested in order get a sense of a program's culture. When choosing a program, review the demographics of the faculty and leadership in order to gain an appreciation of the program's commitment to diversity, and on your interview day, it is at times appropriate to ask specific questions regarding the support for minority applicants and faculty. For applicants considering having a child during fellowship, make sure you understand the parental leave policy and talk with fellows who have had children during their training to gain a better understanding of the process.

\section{Letters of Recommendation}

Since letters of recommendation (LORs) are key tools for programs to gain insight into who you are as a physician and person, it is important to select your letter writers wisely. It is important to ask potential letter writers if they feel comfortable writing you a strong letter; while somewhat rare, there are circumstances in which a potential letter writer may fear that they cannot adequately write a strong letter and may opt out of doing so. The most valuable is a strong letter from someone who knows you well who can adequately describe your accomplishments, potential, and skills in detail. There might be faculty with whom you have had a very close relationship, but they might not be good letter writers. Soliciting opinions from your program leadership or senior residents about who are good letter writers can be helpful. If your program has a tool that provides access to written evaluations, using these written evaluations can help provide insight on who may be a potentially good letter writer; i.e., if you can read that a faculty member has given you glowing evaluations from your time on service together, they may be an excellent person to ask for a letter in the future. It is advisable to meet with your letter writers in person when requesting a LOR. During your meeting, we recommend providing them with your resume, your personal statement, and any points about your work with them that you specifically want them to highlight. For example, an attending with whom you worked on the inpatient service could be tasked with highlighting your bedside manner, professionalism, efficiency, and clinical knowledge, whereas your research mentor could be tasked with highlighting your specific research projects, work ethic, and ability to see a project through to completion. An in-depth discussion 
regarding your fellowship goals and what you are looking for in a fellowship program will also help them write a stronger letter. Also, remember that attending physicians are busy, so be sure to give your letter writers ample time to write and upload your letter. Some residents also feel that it is also not a bad idea to ask for an extra letter in case one of your original letter writers cannot finish your letter in time due to any unforeseen circumstances. ERAS allows you to assign different letters to different programs, an option that can be especially helpful if you know certain faculty might know people in a specific program and their letter may carry more weight at a given institution.

\section{Personal Statement}

Since the personal statement can be easy to write for some and hard to write for others, it is important to get help if you have any concerns about your abilities in order to reduce anxiety and ensure a superior product. The golden rule for personal statements is that it should not harm or "raise any red flags" about your application. Personal statements should not be a re-description of your resume, but rather should provide some insight into your personality and reasons for pursuing gastroenterology. Review your final personal statement to make sure there are no grammatical errors or any controversial statements. It is advisable to have trustworthy mentors and colleagues who are proficient in English writing review your personal statement.

\section{Where to Apply}

The decision of where and how many programs to apply to will depend on an applicant's personal preferences, family situation, career goals, and competitiveness within the match. For example, for those interested in a NIH-funded T32 research track, they may want to focus on academically oriented programs that offer this as a part of fellowship training. Or, for those interested in possibly combining gastroenterology and transplant hepatology training into a three-year program, it is recommended to read the institution's website and talk to program leadership about these opportunities as you are starting to determine which programs will fit your overall fellowship needs and goals. Overall, it is highly recommended to schedule a meeting with your residency program director and the gastroenterology program director in order to obtain an honest assessment of the strength of your application that in turn will help determine how many programs to which you need to apply in order to have a reasonable chance at matching.

\section{Interviews}

Congratulations-you have now made it to interviews. It can be difficult to take time during residency to book hotels, manage scheduling conflicts, and actually travel to the interview site. But, while the "interview trail" can be stressful and exhausting, it is an exceedingly important aspect of the process. One useful principle is that a good interview is essentially a good conversation. Eventually, as a fellow, you will spend a large amount of time with GI faculty, so if you are invited to a program, it means that they already believe you to be well accomplished, and the interviewers primarily want to ascertain if you will be a good fit in the program. Consider practicing with mock interviews with your peers or close faculty before your first interview. While it might sound silly, typing or writing out your answers to specific interview questions that you can read over the night before each interview can sometimes be helpful as well (i.e., "tell me about your research," "why gastroenterology?," "what are you looking for in a program?" are all questions that you should be prepared to answer). During the interview, it is important to create a positive impression on everyone you meet, so be positive, passionate, show interest in the program, and most importantly be yourself. If you include unique strengths and experiences in your application, be sure to mention them and highlight how they made you a better person and physician. Do not forget to be prepared to talk about anything you have written about in your application; make sure that you review your prior research, abstracts, and publications that are listed on your $\mathrm{CV}$, as anything is fair game to be asked about during the interview process. Again, it is essential to "be yourself" and honest in the interview.

\section{Post-interview Communication}

There is no clear consensus regarding post-interview communication. You should respect that some programs will mention during the interview day that they do not want post-interview communication. The key is to not overdo it, make sure your communications are not generic, and highlight specific personalized aspects that you like about the program and/or updates about your scholarly work. It is strongly advised to review and follow ERAS and the NRMP guidelines about the content of communication emails. 


\section{You Did Not Match}

There is always a possibility that one does not match. This is distressing, frustrating, and demoralizing and unfortunately not uncommon; the first author (ND) of this article felt all of these emotions. In this situation, the key is to take a few days off if possible and then reflect, rethink, and restart. You want to carefully evaluate your application for gaps and weaknesses and reach out to mentors and colleagues who will give you honest and genuine advice. You need to revert back to the "modifiable factors" in your application that were discussed in Part I. Since the match results are announced in December and you will need to re-apply in July, you will have roughly 7-8 months to work on bridging the gaps as best as you can. Starting feasible projects with quick turnover time, such as case reports, review articles, and submitting abstracts to upcoming conferences is a winning strategy. You will also need to plan on what to do during your gap year. There is no right answer, but it is important to know what options are available. There are hospitalist jobs that have adequate downtime for working on research projects, dedicated research fellowships, and several non-ACGME accredited subspecialty fellowships such as hepatology, motility, IBD, and nutrition. Nevertheless, if you go the route of a non-ACGME accredited fellowship, this will begin around the time of the next application season, and you will be extremely busy with this new clinical workload. Thus, it is important to make the most of the months between the first match result and the next application season to truly strengthen your resume.

If you are able to improve upon your application, programs will see your ability to not dwell on defeat, and to improve your attractiveness as a candidate and your persistence as strengths. Over these intervening months, reach out and seek mentors and program directors during conferences or networking opportunities, these opportunities show your interest and offer them a chance to get to know you, and demonstrates your perseverance and ultimately your unwavering desire to become a gastroenterologist despite a setback along the way.

\section{Conclusions}

Although there is no "magic rule" to getting into gastroenterology fellowship, in this article we outlined a basic guide on how to become a competitive fellowship applicant. Each applicant has their own unique strengths and weaknesses, and certain other factors, such as finding appropriate helpful and experienced mentors and promoters, achieving scholarly work, and developing a professional network can be helpful.
We hope that this article will help you prepare for your GI fellowship application and we wish you the best of luck!

\section{Take Home Points}

- Gastroenterology is one of the most competitive subspecialties of the fellowship match

- Certain non-modifiable and modifiable factors are important in the application process

- Non-modifiable factors are: USMLE scores, medical school and residency program attended, and visa and IMG status

- Modifiable factors are: scholarly work, letters of recommendation, aspects of the resume, and the development of professionalism, interpersonal skills, and networking

- Successful applicants continue to build and improve upon modifiable factors

- Connecting with and forming relationships with mentors and promoters is imperative for future success

Author's contributions ND and MB were involved in concept design and drafting of the manuscript. EA, KEH, and CRSL wrote critical portions of the manuscript and provided expert opinion. All authors contributed to the manuscript.

Funding None with respect to this manuscript.

\section{Compliance with Ethical Standards}

Conflict of interest The authors report no conflict of interest and have no financial disclosures with respect to this manuscript.

\section{References}

1. Singh S, et al. Updates from the AGA young delegates program. http://agaperspectives.gastro.org. Accessed 17 May 2016.

2. Bilal M, Taleban S, Riegler J, Surawicz C, Feld A. The do's and don'ts of social media: a guide for gastroenterologists. Am J Gastroenterol.. 2019;114:375-376.

3. Day LW, Gonzalez S, Ladd AM, et al. Diversity in gastroenterology in the United States: Where are we now? Where should we go? Gastrointest Endosc.. 2016;83:679-683.

4. Sethi S, Mendoza S, Webb A, Edwards J, Kumar A, Chae S. Addressing gender disparity: increase in female fellowship program leadership increases gender equality in program director and fellow ranks. Gastrointest Endosc.. 2019;89:AB61-AB62.

Publisher's Note Springer Nature remains neutral with regard to jurisdictional claims in published maps and institutional affiliations. 